\title{
ALMOST COMPLETE TILTING MODULES
}

\author{
DIETER HAPPEL AND LUISE UNGER
}

(Communicated by Donald Passman)

Dedicated to Prof. H. Tachikawa on the occasion of his $60^{\text {th }}$ birthday

\begin{abstract}
Let $A$ be a finite-dimensional hereditary algebra over an algebraically closed field. All modules will be finite-dimensional left $A$-modules. We are concerned with partial tilting modules which can be completed to a tilting module by one indecomposable module which will be called a complement. As a main result we show that such a partial tilting module allows (up to isomorphism) at most two complements and there are two such complements if and only if the partial tilting module is sincere.
\end{abstract}

Let $A$ be a basic and connected finite-dimensional hereditary algebra over an algebraically closed field $k$ (i.e., $A$ is given as a path algebra $k \vec{\Delta}$ of a finite and connected quiver $\vec{\Delta}$ without oriented cycles). We denote by $A$-mod the category of finitely generated left $A$-modules. We assume that $A$ has $n$ isomorphism classes of simple modules. A partial tilting module ${ }_{A} M$ (i.e., ${ }_{A} M$ satisfies $\left.\operatorname{Ext}_{A}^{1}(M, M)=0\right)$ is called an almost complete tilting module, if ${ }_{A} M$ has $n-1$ nonisomorphic indecomposable direct summands. An indecomposable A-module ${ }_{A} X$ is called a complement to ${ }_{A} M$, if $M \oplus X$ is an $A$-tilting module (i.e. $\operatorname{Ext}_{A}^{1}(M \oplus X, M \oplus X)=0$ and $X$ is not isomorphic to a direct summand of $M$ ). Tilting modules have been found to be very useful in the representation theory of finite-dimensional algebras $($ see $[\mathrm{H}]$ and the references given there). Usually we will assume that our tilting modules are multiplicityfree or equivalently that $\operatorname{End}_{A} T$ is a basic algebra whenever ${ }_{A} T$ is a tilting module. It was shown in [HR] (see also [B]) that there is always a complement to ${ }_{A} M$, and it has been proved in [RS] and [U], that there are at most two nonisomorphic complements.

The aim of this article is to prove

Theorem. An almost complete tilting module ${ }_{A} M$ has exactly two nonisomorphic complements if and only if ${ }_{A} M$ is sincere.

Received by the editors February 21, 1989.

1980 Mathematics Subject Classification (1985 Revision). Primary 16A46, 16A64.

Key words and phrases. Tilting modules, complements, hereditary algebras. 
Recall that a module ${ }_{A} N$ is called sincere, if $\operatorname{Hom}_{A}(P, N) \neq 0$ for all indecomposable projective $A$-modules $P$.

This generalizes a result observed in [U], where this was shown in the case $n=3$ and the additional assumption that all indecomposable direct summands of $M$ are regular $A$-modules.

Essential for the proof is the following result basically contained in 2.1 of [U].

Theorem. Let ${ }_{A} M$ be an almost complete tilting module, and let $X$ and $Y$ be two nonisomorphic complements to ${ }_{A} M$. Assume without loss of generality, that $\operatorname{Ext}_{A}^{1}(Y, X) \neq 0$. Then there is a nonsplit exact sequence

$$
0 \rightarrow X \rightarrow E \rightarrow Y \rightarrow 0
$$

with $E \in$ add $M$ (where as usual add $M$ denotes the additive category generated by the direct summands of $M$ ).

From this theorem we deduce the following immediate consequences from [RS]:

Corollary. There are at most two nonisomorphic complements to an almost complete tilting module ${ }_{A} M$.

Corollary. If the almost complete tilting module ${ }_{A} M$ is not sincere, then there is exactly (up to isomorphism) one complement to ${ }_{A} M$.

Note that this is one implication of the first theorem.

In the first section we will prove the second theorem and its consequences. In the second section we show the missing implication of the first theorem.

In addition we determine in the last section the unique complement to an almost complete tilting module which is not sincere by constructing two exact sequences containing the complement as middle term.

\section{UNIVERSAL SEQUENCES FOR DISTINCT COMPLEMENTS TO ${ }_{A} M$}

We will keep the notations from the introduction, and from now on, we will consider modules only up to isomorphism.

Let $X$ and $Y$ be $A$-modules with $0 \neq r=\operatorname{dim}_{k} \operatorname{Ext}_{A}^{1}(Y, X)$. Using the same arguments as in [B] there exists an exact sequence $0 \rightarrow X \rightarrow E \rightarrow Y^{r} \rightarrow 0$ such that the connecting homomorphism $\delta: \operatorname{Hom}_{A}\left(Y, Y^{r}\right) \rightarrow \operatorname{Ext}_{A}^{1}(Y, X)$ is surjective. An exact sequence with this property will be called universal. Note that such a sequence is uniquely determined (up to isomorphism) if $\operatorname{Hom}_{A}(Y, Y)=$ $k$.

1.1 Theorem. Let ${ }_{A} M$ be an almost complete tilting module, and let $X$ and $Y$ be two complements to $M$. Assume, that $\operatorname{Ext}_{A}^{1}(Y, X) \neq 0$. Then there exists a universal sequence

$$
0 \rightarrow X \rightarrow E \rightarrow Y \rightarrow 0
$$

with $E \in$ add $M$. 
Proof. Let $r=\operatorname{dim}_{k} \operatorname{Ext}_{A}^{1}(Y, X)$ and consider the universal sequence

$$
\eta: 0 \rightarrow X \stackrel{\mu}{\longrightarrow} E \stackrel{\pi}{\longrightarrow} Y^{r} \rightarrow 0 \text {. }
$$

It is easy to prove, that $Y$ is not a direct summand of $E$. We will prove first that $E$ belongs to add $M$. Since $\eta$ is universal, we obtain that $\operatorname{Ext}_{A}^{1}(Y, E)=$ 0 , and since $X$ and $Y$ are complements to $M$, we get that $\operatorname{Ext}_{A}^{1}(M, E)=0=$ $\operatorname{Ext}_{A}^{1}(E, M)$. Then $E$ is generated by $M \oplus Y$.

For $s, t \in \mathbb{N}$ consider the exact sequence

$$
0 \rightarrow Y^{t} \stackrel{f}{\longrightarrow} M^{s} \oplus Y^{t} \stackrel{g}{\longrightarrow} M^{s} \rightarrow 0
$$

where $f$ is the canonical inclusion and $g$ is the canonical projection. We denote by $\epsilon=\left(\epsilon_{1}, \epsilon_{2}\right)^{T}$ a surjective map from $M^{s} \oplus Y^{t}$ to $E$, which exists by the considerations above. Let $\epsilon_{2}$ be given by $\left(\epsilon_{21}, \ldots, \epsilon_{2 t}\right)$. Let $\pi=\left(\pi_{1}, \cdots, \pi_{r}\right)$. By construction, $\epsilon_{2 i} \pi_{j}$ is a proper factorization for all $1 \leq i \leq t$ and $1 \leq j \leq r$. Note that we compose maps from left to right. Since the endomorphism ring of $Y$ is the ground field $k$, we obtain that $\epsilon_{2} \pi=0$. Thus there is a map $\phi$ from $Y^{t}$ to $X$ with $f \epsilon=\phi \mu$, and therefore there is a map $\psi$ from $M^{s}$ to $Y^{r}$ with $g \psi=\epsilon \pi$, in particular, $\psi$ is surjective, and so $Y$ is generated by $M$. This implies that $\operatorname{Ext}_{A}^{1}(X, Y)=0$, thus $\operatorname{Ext}_{A}^{1}(X, E)=0=\operatorname{Ext}_{A}^{1}(E, E)$. But then also $\operatorname{Ext}_{A}^{1}(E, Y)=0$ Thus $E \oplus M \oplus Y$ is a tilting module, and since $Y$ is not a direct summand of $E$ we obtain that $E \in$ add $M$.

It remains to show that $\operatorname{Ext}_{A}^{1}(Y, X)=k$. Applying $\operatorname{Hom}_{A}(-, X)$ to the sequence $\eta$ yields the following long exact sequence:

$$
\operatorname{Hom}_{A}(E, X) \rightarrow \operatorname{Hom}_{A}(X, X) \rightarrow \operatorname{Ext}_{A}^{1}\left(Y^{r}, X\right) \rightarrow 0 .
$$

We have used that $\operatorname{Ext}_{A}^{1}(E, X)=0$, for $M \oplus X$ is a tilting module. Now $\operatorname{Hom}_{A}(X, X)=k$ shows that $r=1$.

We also point out that it is easily seen that $\operatorname{Hom}_{A}(Y, X)=0$.

1.2 Corollary. There are at most two complements to an almost complete tilting module ${ }_{A} M$.

Proof. Let $X$ and $Y$ be two complements to $M$, and let $Z$ be a third complement. We may assume without loss of generality, that $\operatorname{Ext}_{A}^{1}(Y, X) \neq 0$. We consider the universal sequence $0 \rightarrow X \rightarrow E \rightarrow Y \rightarrow 0$. Applying $\operatorname{Hom}_{A}(Z,-)$ and $\operatorname{Hom}_{A}(-, Z)$ yields that $\operatorname{Ext}_{A}^{1}(Z, Y)=0=\operatorname{Ext}_{A}^{1}(X, Z)$. Since by assumption $Z$ and $X$ are not isomorphic we conclude that $\operatorname{Ext}_{A}^{1}(Z, X) \neq 0$. We then have a universal sequence $0 \rightarrow X \rightarrow E^{\prime} \rightarrow Z \rightarrow 0$ with $E^{\prime} \in$ add $M$. Applying $\operatorname{Hom}_{A}(Y,-)$ shows that $\operatorname{Ext}_{A}^{1}(Y, Z)=0$, hence $M \oplus Y \oplus Z$ is a tilting module. This is a contradiction, since an $A$-tilting module has precisely $n$ nonisomorphic indecomposable direct summands.

1.3. As a second consequence of the theorem we obtain one implication of our main result mentioned in the introduction. 
Corollary. If the almost complete tilting module ${ }_{A} M$ is not sincere, then there is exactly one complement to $M$.

Proof. Since $M$ is not sincere, there exists a primitive idempotent $\omega \in A$, such that $\operatorname{Hom}_{A}(A \omega, M)=0$. Assume that $X$ and $Y$ are two complements to $M$. We may assume without loss of generality that $\operatorname{Ext}_{A}^{1}(Y, X) \neq 0$. Consider the universal sequence $0 \rightarrow X \rightarrow E \rightarrow Y \rightarrow 0$ with $E \in$ add $M$. Applying $\operatorname{Hom}_{A}(A \omega,-)$ shows that $\operatorname{Hom}_{A}(A \omega, X)=0=\operatorname{Hom}_{A}(A \omega, Y)$. Then $M \oplus X$ is a nonsincere tilting module, an absurdity.

\section{COMPLEMENTS TO SINCERE ALMOST COMPLETE TILTING MODULES}

In order to prove the second implication, we only have to prove that if an almost complete tilting module ${ }_{A} M$ is sincere, then there are at least two different complements to $M$.

2.1. Let ${ }_{A} N$ be an $A$-module satisfying $\operatorname{End}_{A} N=k$ and $\operatorname{Ext}_{A}^{1}(N, N)=0$. We denote the full subcategory of all $A$-modules ${ }_{A} X$ satisfying $\operatorname{Hom}_{A}(N, X)=0$ and $\operatorname{Ext}_{A}^{1}(N, X)=0$ by $N^{\perp}$, and the full subcategory of all $A$-modules ${ }_{A} Y$ satisfying $\operatorname{Hom}_{A}(Y, N)=0$ and $\operatorname{Ext}_{A}^{1}(Y, N)=0$ by ${ }^{\perp} N$. It has been proved in [GL] (compare [H] and [S]), that $N^{\perp}$ and ${ }^{\perp} N$ are equivalent to module categories $A_{o}$-mod and $A_{o}^{\prime}$-mod, where $A_{o}$ and $A_{o}^{\prime}$ are finite-dimensional hereditary $k$-algebras with $n-1$ simple modules.

Let $L=\bigoplus_{i=1}^{r} N_{i}$ be a direct sum decomposition of a partial tilting module $L$ into indecomposable direct summands. We then define $L^{\perp}$ and ${ }^{\perp} L$ to be the intersection of the $N_{i}^{\perp}$ respectively ${ }^{\perp} N_{i}$. One can easily prove by induction, that $L^{\perp}$ and ${ }^{\perp} L$ are equivalent to hereditary module categories $B$-mod and $B^{\prime}$-mod, where $B$ and $B^{\prime}$ have $n-r$ simple modules. In particular if ${ }_{A} M$ is an almost complete tilting module, then $M^{\perp}$ and ${ }^{\perp} M$ are generated as additive categories by indecomposable modules $Z$ and $Z^{\prime}$ respectively, and clearly $\operatorname{Ext}_{A}^{1}(Z, Z)=0=\operatorname{Ext}_{A}^{1}\left(Z^{\prime}, Z^{\prime}\right)$.

2.2. It is easy to prove, that if $L$ is a sincere $A$-module with $\operatorname{Ext}_{A}^{1}(L, L)=$ 0 , then the restriction of the Auslander-Reiten translation functor $\left.\tau\right|_{\perp_{L}}$ is an equivalence from ${ }^{\perp} L$ to $L^{\perp}$, and $\left.\tau^{-}\right|_{L^{\perp}}$ is an equivalence from $L^{\perp}$ to ${ }^{\perp} L$. (For a proof we refer to $[\mathrm{S}]$ or $[\mathrm{U}]$.)

Hence, if ${ }_{A} M$ is a sincere almost complete $A$-tilting module, and if $M^{\perp}$ is generated by the indecomposable module ${ }_{A} Z$, then ${ }^{\perp} M$ is generated by $\tau^{-} Z$. In particular, if $X$ and $Y$ are two complements to $M$ with $X \in{ }^{\perp} M$ and $Y \in M^{\perp}$, then the universal sequence from 1.1. is an Auslander-Reiten sequence.

The following is the missing implication of the first theorem of the introduction. 
2.3 Proposition. If ${ }_{A} M$ is a sincere almost complete tilting module, then there are two complements to ${ }_{A} M$.

Proof. Let $M^{\perp}$ be generated by $Z$ and let ${ }^{\perp} M$ be generated by $\tau^{-} Z$. Assume that there is exactly one complement to $M$.

We first consider the case that $X$ belongs to $M^{\perp}$, hence $X \simeq Z$. Since by assumption, $\tau^{-} Z$ is not a complement to $M$, we have that $\operatorname{Ext}_{A}^{1}\left(M, \tau^{-} Z\right) \neq$ 0 . Let $0 \rightarrow \tau^{-} Z \rightarrow Y \rightarrow M^{t} \rightarrow 0$ be the universal sequence. Applying $\operatorname{Hom}_{A}(M,-)$ and $\operatorname{Hom}_{A}(-, M)$ yields that $\operatorname{Ext}_{A}^{1}(M, Y)=0=\operatorname{Ext}_{A}^{1}(Y, M)$. Applying $\operatorname{Hom}_{A}(-, Y)$ to the sequence above, we obtain, that $\operatorname{Ext}_{A}^{1}(Y, Y)$ and $\operatorname{Ext}_{A}^{1}\left(\tau^{-} Z, Y\right)$ are isomorphic. Applying finally $\operatorname{Hom}_{A}(-, Z)$ shows that $\operatorname{Hom}_{A}(Y, Z)=0=\operatorname{Ext}_{A}^{1}\left(\tau^{-} Z, Y\right)$. Then $M \oplus Y$ is a tilting module, and by assumption, $Z$ is isomorphic to a direct summand of $Y$. But then $0 \neq$ $\operatorname{Hom}_{A}(Y, Z)=\operatorname{Hom}_{A}\left(\tau^{-} Z, Z\right) \simeq D \operatorname{Ext}_{A}^{1}(Z, Z)$ (where $D$ is the standard duality), an absurdity.

Dually we can disprove the assumption, that $X$ belongs to ${ }^{\perp} M$.

Hence, $X$ neither belongs to $M^{\perp}$ nor to ${ }^{\perp} M$, implying that $\operatorname{Ext}_{A}^{1}(Z, M)$ $\neq 0$ and $\operatorname{Ext}_{A}^{1}\left(M, \tau^{-} Z\right) \neq 0$.

Consider the universal sequences

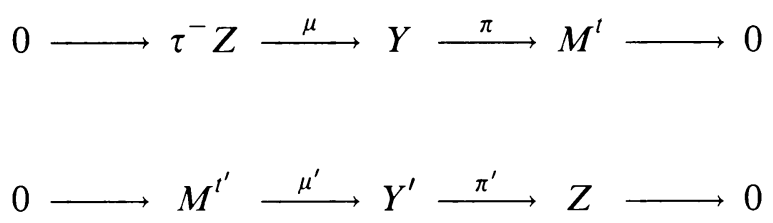

The same arguments as above show, that $M \oplus Y$ and $M \oplus Y^{\prime}$ are tilting modules, hence $X$ is isomorphic to a direct summand of $Y$ and of $Y^{\prime}$. Let $\mu_{1}$ be the component of $\mu$ mapping $\tau^{-} Z$ to $X$. By construction we infer that $\mu_{1} \neq 0$.

Since $\operatorname{Ext}_{A}^{1}(Z, Z)=0=\operatorname{Hom}_{A}\left(\tau^{-} Z, Z\right)$, we obtain a map $f$ from $\tau^{-} Z$ to $M^{t^{\prime}}$ with $\mu_{1}=f \mu^{\prime}$. Since $\tau^{-} Z \in{ }^{\perp} M$ we infer that $f=0$, thus $\mu_{1}=0$, a contradiction.

\section{THE COMPLEMENT TO A NON-SINCERE ALMOST COMPLETE TILTING MODULE}

In this section we will assume, that ${ }_{A} M$ is a nonsincere almost complete tilting module, say $\operatorname{Hom}_{A}(A \omega, M)=0$ for some primitive idempotent $\omega$ of $A$. Denote by $R$ the radical of $A \omega$, and by $S(\omega)=A \omega / R$ the top of $A \omega$. So we have an exact sequence $\eta: 0 \rightarrow R \rightarrow A \omega \rightarrow S(\omega) \rightarrow 0$. Let $B=A / A \omega A$. So $M$ can be considered as a $B$-tilting module. Note that $B$ is hereditary. Obviously, $M^{\perp}$ is generated by the injective A-module $D(\omega A)$, and ${ }^{\perp} M$ is generated by $A \omega$. We wish to describe the unique complement $X$ to $M$.

We distinguish the following three cases. Either $S(\omega)$ is injective or $S(\omega)$ is projective. And finally the case that $S(\omega)$ is neither projective nor injective. 
3.1. Assume that $S(\omega)$ is injective. Then $A=B[R]$ is the one-point extension algebra of $B$ by the radical of $A \omega$. This is by definition the finite-dimensional $k$-algebra

with multiplication

$$
B[R]=\left(\begin{array}{ll}
B & R \\
0 & k
\end{array}\right)
$$

$$
\left(\begin{array}{ll}
b & r \\
0 & \lambda
\end{array}\right)\left(\begin{array}{cc}
b^{\prime} & r^{\prime} \\
0 & \lambda^{\prime}
\end{array}\right)=\left(\begin{array}{cc}
b b^{\prime} & b r^{\prime}+r \lambda^{\prime} \\
0 & \lambda \lambda^{\prime}
\end{array}\right)
$$

where $b, b^{\prime} \in B, r, r^{\prime} \in R$ and $\lambda, \lambda^{\prime} \in k$. The following generalizes a result of [Ri2].

Lemma. $R$ is a summand of $M$ if and only if $X=A \omega \in{ }^{\perp} M$. In particular, if $R$ is not a summand of $M$, then the complement $X$ satisfies: $\operatorname{Hom}_{A}(M, X) \neq 0$ and $\operatorname{Hom}_{A}(X, M) \neq 0$.

Proof. Applying $\operatorname{Hom}_{A}(-, M)$ to the sequence $\eta$, we obtain, that $\operatorname{Hom}_{A}(R, M)$ and $\operatorname{Ext}_{A}^{1}(S(\omega), M)$ are isomorphic. Since $M$ is an almost complete tilting module, we infer that $\operatorname{Hom}_{A}(R, M)$ is not zero, hence $S(\omega)$ is not a complement to $M$. In particular, $X \notin M^{\perp}$.

Assume that $R$ is a direct summand of $M$. The application of $\operatorname{Hom}_{A}(M,-)$ and $\operatorname{Hom}_{A}(-, M)$ to $\eta$ now shows, that $\operatorname{Ext}_{A}^{1}(M, A \omega)=0$ and $\operatorname{Ext}_{A}^{1}(A \omega, M)$ $=0$. Since $A \omega$ is projective we conclude that $A \omega=X$ is the complement to $M$.

Assume now, that $X$ is contained in ${ }^{\perp} M$, i.e. $X=A \omega$. Then we have that $\operatorname{Ext}_{A}^{1}(M, R)=0$ and $\operatorname{Ext}_{A}^{1}(R, M)=0$. Therefore $R$ is a direct summand of $M$.

In particular this shows, that if all direct summands of $M$ are regular, then the complement $X$ will also be regular.

3.2. Assume that $S(\omega)$ is projective. Using the dual concept of one-point coextension algebras (compare [Ril]) one can show that $D(\omega A) / S(\omega)$ is a direct summand of $M$ if and only if $X=D(\omega A) \in M^{\perp}$. In particular, if $D(\omega A) / S(\omega)$ is not a direct summand of $M$, then the complement $X$ satisfies: $\operatorname{Hom}_{A}(M, X) \neq 0$ and $\operatorname{Hom}_{A}(X, M) \neq 0$.

3.3. Assume now that $S(\omega)$ is neither injective nor projective. In this case we have exact sequences

$$
0 \rightarrow P \rightarrow A \omega \rightarrow S(\omega) \rightarrow 0
$$

where $0 \neq P$ is $A$-projective and

$$
0 \rightarrow S(\omega) \rightarrow D(\omega A) \rightarrow I \rightarrow 0
$$

where $0 \neq I$ is $A$-injective. Obviously, $\operatorname{Hom}_{A}(P, M) \neq 0$, showing that $\operatorname{Ext}_{A}^{1}(S(\omega), M) \neq 0$. This implies, that $\operatorname{Ext}_{A}^{1}(D(\omega A), M) \neq 0$, thus $X \notin M^{\perp}$. Dually, since $\operatorname{Hom}_{A}(M, I) \neq 0$, we get that $\operatorname{Ext}_{A}^{1}(M, S(\omega)) \neq 0$. This yields that $\operatorname{Ext}_{A}^{1}(M, A \omega) \neq 0$, and therefore $X \notin{ }^{\perp} M$. 
3.4. Summarizing our previous considerations, we obtain

Proposition. Let ${ }_{A} M$ be a nonsincere almost complete tilting module with $\operatorname{Hom}_{A}(A \omega, M)=0$ for some primitive idempotent $\omega \in A$. Let $R=\operatorname{rad} A \omega$, $R^{\prime}=D(\omega A) / S(\omega)$ and let $B=A / A \omega A$. Then the unique complement $X$ to $M$ satisfies $\operatorname{Hom}_{A}(M, X) \neq 0$ and $\operatorname{Hom}_{A}(X, M) \neq 0$, unless we have one of the following two situations:

(1) $A$ is the one-point extension algebra $B[R]$, and $R$ is a direct summand of $M$. In this case $X=A \omega$.

(2) $A$ is the one-point coextension algebra $\left[R^{\prime}\right] B$, and $R^{\prime}$ is a direct summand of $M$. In this case $X=D(\omega A)$.

It is easily seen that there exist $M^{\prime}, M^{\prime \prime} \in$ add $M$ and exact sequences

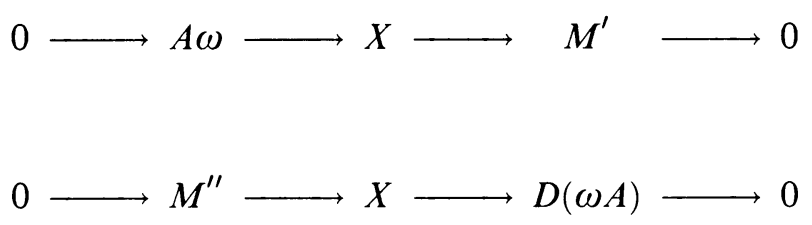

where $X$ is the unique complement to the nonsincere almost complete tilting module ${ }_{A} M$. We construct the first sequence. The second can be obtained by dual considerations. In fact, let $r=\operatorname{dim}_{k} \operatorname{Ext}_{A}^{1}(M, A \omega)$. Let $0 \rightarrow A \omega \rightarrow E \rightarrow$ $M^{r} \rightarrow 0$ be the universal sequence. As before we infer that $E \oplus M$ is a tilting module. Since $\operatorname{Hom}(A \omega, E)=k$ there is a unique indecomposable summand $X$ of $E$ such that $\operatorname{Hom}(A \omega, X) \neq 0$. Thus $E=X \oplus \widetilde{M}$, with $\widetilde{M} \in \operatorname{add} M$. Let $\mu$ be a nonzero map from $A \omega$ to $X$ which must be injective. Set $M^{\prime}=\operatorname{coker} \mu$. Then it is easily seen that $M^{\prime}$ belongs to add $M$.

We have shown before, that at least one of the sequences is nonsplit and that both sequences are nonsplit, unless we are in the situations (1) or (2) described in the proposition above.

\section{REFERENCES}

[B] K. Bongartz, Tilted algebras, Proceedings of ICRA III, Springer Lecture Notes vol. 903 Heidelberg, 1981, 26-38.

[GL] W. Geigle and H. Lenzing, Perpendicular categories with applications to representations and sheaves, preprint.

[H] D. Happel, Triangulated categories and the representation theory of finite-dimensional algebras, LMS Lecture Note Series vol. 110 Cambridge, 1988.

[HR] D. Happel and C.M. Ringel, Tilted algebras, Trans. Amer. Math. Soc. 274 (1982), 399-443.

[Ri1] C.M. Ringel, Tame algehras and integral quadratic forms, Springer Lecture Notes vol. 1099 Heidelberg, 1984.

[Ri2] C.M. Ringel, The regular components of the Auslander-Reiten quiver of a tilted algehra, Chin. Ann. Math. Ser. B 9 (1988), 1-18. 
[RS] C. Riedtmann and A. Schofield, On open orbits and their complements, preprint.

[S] A. Schofield, Generic representations of quivers, preprint.

[U] L. Unger, Schur modules over wild path algebras with three simple modules, (to appear in J. of Pure and Applied Alg.).

Fakultät für Mathematik, Universität Bielefeld, Postfach 8640, 4800 Bielefeld 1, Federal Republic of Germany

Fachbereich Mathematik, Universität Paderborn, Warburgerstr.100， 4790 Paderborn, Federal Republic of Germany 